BULL. AUSTRAL. MATH. SOC.

$54 A 25,54 F 05,02 K 05,06 A 65$

VOL. 25 (1982), 425-431.

\title{
A CHARACTERIZATION OF THE EXISTENCE OF A SOUSLIN LINE
}

NOBUYUKI KEMOTO

\begin{abstract}
The main purpose of this paper is to show that there exists a Souslin line if and only if there exists a countable chain condition space which is not weak-separable but has a generic $\pi$-base. If $I$ is the closure of the isolated points in a space $X$, then $X$ is said to be weak-separable if a first category set is dense in $X-I$. A $\pi$-base $\underline{B}$ is said to be generic if, whenever a member of $\underline{\underline{B}}$ is included in the disjoint union of members of $\underline{\underline{B}}$, it is included in one of them.
\end{abstract}

Miller [4], showed that the equivalence of the existence of a Souslin line and the existence of a Souslin tree. Furthermore Tall [7] obtained a topological condition of the existence of a Souslin line without mentioning the orderability. In this paper we shall give a simpler equivalence condition for the existence of a Souslin line.

A Souslin line is a linearly ordered countable chain condition topological space which is not separable. A tree is a partial ordered set in which for each element, the set of its predecessors is well ordered. A tree $T$ is said to be Souslin if $T$ is uncountable for which every chain and every antichain are countable.

It is well known that the existence of a Souslin line can not be proved or refuted from the usual axioms of set theory; see Solovay and Tennenbaum [6].

Received 4 January 1982. The author wishes to express his thanks to the referee for observing his paper. 
DEFINITION 1. Let $X$ be a topological space. A $\pi$-base $\underline{\underline{B}}$ for $X$ is a collection of open sets of $X$ such that for each non-empty open set there exists a member of $\underline{\underline{B}}$ which is included in it. A $\pi$-base $\underline{\underline{B}}$ is said to be generic if, whenever a member of $\underline{\underline{B}}$ is included in the disjoint union of $\underline{\underline{B}}$, it is included in one of them.

Next we shall give a new class of topological spaces.

DEFINITION 2. A topological space $X$ is said to be weak-separable if a first category set is dense in $X-I$, where $I$ is the closure of the isolated points in $X$.

Clearly, separable space is weak-separable. But the converse is not true; see the example on $p$.

LEMMA 1. Let $I=(u, v)$ be a separable open interval without isolated points of a linearly ordered topological space; then $I$ has a generic $\pi$-base for $I$.

Proof. Let $A=\left\{a_{k}: k<\omega_{0}\right\}$ be a dense subset of $I$. By induction on $\omega_{0}$, we will construct $\stackrel{B}{\Rightarrow}_{m}$ of a finite family of disjoint intervals and a finite subset $C_{m}$ of $A$ for each $m<\omega_{0}$. First let $\underline{\mathrm{B}}_{0}=\{I\}$ and $C_{0}=\left\{a_{0}\right\}$. Assume we have constructed $\stackrel{\mathrm{B}}{m}_{m}=\left\{\left(x_{n}, y_{n}\right): n<m_{0}\right\}$ and $C_{m}$, where $x_{n}, y_{n}$ are elements of $A \cup\{u, v\}$ for each $n<m_{0}$. For each $n<m_{0}$, let $k_{n}$ be the least $k$ such that $a_{k}$ is in $\left(x_{n}, y_{n}\right)$. Then put

$$
c_{m+1}=\left\{a_{k_{n}}: n<m_{0}\right\}
$$

and

$$
\stackrel{\mathrm{B}}{=}_{m+1}=\left\{\left(x_{n}, a_{k_{n}}\right): n<m_{0}\right\} \cup\left\{\left(a_{k_{n}}, y_{n}\right): n<m_{0}\right\}
$$

Then easily we can prove that $\underset{m<\omega_{0}}{U} \stackrel{B}{\Rightarrow}$ is a generic $\pi$-base for $I$.

THEOREM 1. A SOuslin line is a countable chain condition space which is not weak-separable but has a generic $\pi$-base.

Proof. Let $S$ be a Souslin line. $S$ has a countable chain condition 
by definition. Since $S$ is linear and has countable chain condition, the isolated points of $S$ are countable, and every nowhere dense subset of $S$ is separable. Thus, since $S$ is not separable, $S$ is not weak-separable.

Next let $\underline{\underline{I}}=\{\{p\}: p$ is an isolated point of $S\}$, and $\underline{\underline{S}}=\left\{I_{n}: n<\omega_{0}\right\}$ be a maximal family of separable open intervals of $S$. Then, by Lemma 1, there exists a generic $\pi$-base $\underline{\underline{B}}\left(I_{n}\right)$ for $I_{n}$ for each $n<\omega_{0}$. Next, by induction on $\omega_{I}$, we will construct ${ }_{-}=\alpha$ of pairwise disjoint open intervals of $S$ and countable subsets $C_{\alpha}$ and $D_{\alpha}$ of $S$ for each $\alpha<\omega_{1}$. First, let $C_{0}=\varnothing$ and let $\underline{B}_{0}=\left\{\left(x_{n}, y_{n}\right): n<\omega_{0}\right\}$ be a maximal family of pairwise disjoint open intervals of $S$ such that each member of $B_{0}$ is disjoint from $(U I) \cup(U \subseteq)$. Furthermore for each $n<\omega_{0}$, take $z_{n} \in\left(x_{n}, y_{n}\right)$, and put $D_{0}=\left\{x_{n}, y_{n}, z_{n}: n<\omega_{0}\right\}$. Next assume that we have constructed $C_{\beta}, D_{\beta}$ and ${ }_{-\beta}$ for each $\beta<\alpha$. Let $C_{\alpha}=\underset{\beta<\alpha}{\cup} D_{\beta}$ and $\underline{B}_{\alpha}=\left\{\left(x_{n}, y_{n}\right\}: n<\omega_{0}\right\}$ be a maximal family of pairwise disjoint open intervals of $S$ such that each member of ${ }_{=}^{B} \alpha$ is disjoint from $(U \underline{\underline{I}}) \cup(U \underline{\underline{\underline{S}}}) \cup C_{\alpha}$. Furthermore, for each $n<\omega_{0}$, take $z_{n} \in\left(x_{n}, y_{n}\right)$, and put $D_{\alpha}=\left\{x_{n}, y_{n}, z_{n}: n<\omega_{0}\right\}$.

$\operatorname{CLAIM} 1 . \quad S=\overline{U \underline{I}} \cup \overline{U S} \cup\left(\underset{\alpha<\omega_{1}}{\cup} \overline{C_{\alpha}}\right)$.

To prove this we assume $p \notin \overline{U I} \cup \overline{U S} \cup\left(\underset{\alpha<\omega_{1}}{U} \overline{C_{\alpha}}\right)$. Then for each $\alpha<\omega_{1}$, there exists a member $I_{\alpha}$ of $\underline{B}_{\alpha}$ such that $p \in I_{\alpha}$. Since $I_{\alpha+1} \subset I_{\alpha}$ and by the construction, there exists a non-empty open interval $J_{\alpha}$ included in $I_{\alpha}-I_{\alpha+1}$ for each $\alpha<\omega_{1}$. Hence $\left\{J_{\alpha}: \alpha<\omega_{1}\right\}$ is an uncountable family of pairwise disjoint open intervals, which is a contradiction.

CLAIM 2. $\underline{\underline{B}}=U\left\{\underline{\underline{B}}\left(I_{n}\right): I_{n} \in \underline{\underline{S}}\right\} \cup \underline{\underline{I}} \cup \cup\left\{\underline{B}_{-\alpha}: \alpha<\omega_{1}\right\}$ is a $\pi$-base for $S$.

To prove this let $(x, y)$ be a non-empty interval of $S$. If $(x, y)$ 
has an isolated point $p$ then $\{p\} \subset(x, y)$ and $\{p\} \in I$. If $(x, y)$ has no isolated point and $(x, y) \cap I_{n} \neq \varnothing$ for some $I_{n} \in \underline{\underline{S}}$, then there exists an $I \in \underset{B}{\mathrm{~B}}\left(I_{n}\right)$ such that $I \subset(x, y) \cap I_{n}$, because $\mathrm{B}\left(I_{n}\right)$ is a $\pi$-base for $I_{n}$. Let $(x, y)$ has no isolated point and $(x, y) \cap(\cup \underline{\underline{S}})=\phi$. Take $a, b \in S$ such that $x<a<b<y$, then there exists an $\alpha<\omega_{1}$ such that $a, b \in \overline{C_{\alpha}}$. By the maximality of $\underline{S}$, $(a, b)-\bar{C}_{\alpha} \neq A$. Then by the construction of $B_{=\alpha}$, there exists an $I \in \underline{B}_{\alpha}$ such that $I \subset(a, b)-\overline{C_{\alpha}}$. Hence $I \subset(x, y)$. Thus $\underline{B}$ is a $\pi$-base for $S$.

Also one can easily prove the genericity of $\underline{B}$.

This completes the proof.

REMARK. If there exists a Souslin line $S$, then one can construct a connected Souslin line $S^{*}$; see Kunen [2]. Since a connected linearly ordered space is locally connected, all connected open subsets of $S^{*}$ are generic $\pi$-bases for $S^{*}$.

THEOREM 2. If $X$ is a countable chain condition space which is not weak-separable but has a generic $\pi$-base $\underline{\underline{\mathrm{G}}}$, then $\underline{\mathrm{G}}$, partially ordered by reverse inclusion, contains a soustin tree.

Proof. Since $X$ is a countable chain condition we can assume without loss of generality that $X$ has no isolated point. By induction, for each $\alpha<\omega_{1}$, we choose $T_{\alpha} \subset \underline{\underline{G}}$ as follows. Let $T_{0}$ be a maximal family of infinite pairwise disjoint members of $\underline{E}$. Having chosen $\left\{T_{\beta}: B<\alpha\right\}$, define $\underline{G}=\left\{g \in \underline{\underline{G}}: g \subset \bigcap_{\beta<\alpha}\left(U T_{\beta}\right)\right\}$ and $T_{\alpha}^{;}$to be a maximal family of infinite pairwise disjoint members of $\mathrm{G}_{\alpha}$. For $g^{\prime} \in T_{\alpha}^{\prime}$, let $\mathrm{G}_{\alpha}\left(g^{\prime}\right)=\left\{g \in \underline{G}: g \subset g^{\prime}\right\}$ and $T_{\alpha}\left(g^{*}\right)$ is a maximal family of infinite pairwise disjoint members of $G_{-\alpha}\left(g^{\prime}\right)$. Finally let $T_{\alpha}=\left\{T_{\alpha}\left(g^{\prime}\right): g^{\prime} \in T_{\alpha}^{\prime}\right\}$. Since $\underline{\underline{G}}$ is generic, $T=\underset{\alpha<\omega_{1}}{U} T_{\alpha}$, ordered by reverse inclusion, is a tree, and $X$ is a countable chain condition, and by construction, each chain and each antichain are countable. To see that $T$ 
is uncountable, we will show that $X-\operatorname{int}\left(\bigcap_{\beta<\alpha} U T_{\beta}\right)$ has a dense first category set for each $\alpha<\omega_{1}$. Then, since $X$ is not weak-separable, $\underline{G}_{\alpha} \neq \varnothing$ and thus $T_{\alpha} \neq \varnothing$. Hence $T$ is uncountable. Clearly $X-\operatorname{int}\left(U T_{0}\right)=X-\left(U T_{0}\right)$ is nowhere dense. Assume that $X$ - int $\left(\bigcap_{\beta<\alpha} \cup T_{\beta}\right)$ has a dense first category set. By the maximality of $T_{\alpha}^{\prime}$, $\operatorname{int}\left(\bigcap_{\beta<\alpha} U T_{\beta}\right)-\left(U T_{\alpha}^{\prime}\right)$ is nowhere dense. Also $g^{\prime}-\left(U T_{\alpha}\left(g^{\prime}\right)\right)$ is nowhere dense for each $g^{\prime} \in T_{\alpha}$. Since

$$
\begin{aligned}
X-\left(\operatorname{int}\left(\bigcap_{\beta<\alpha+1} U T_{\beta}\right)\right) & =\left(X-\left(\operatorname{int}\left(\bigcap_{\beta<\alpha} U T_{\beta}\right)\right)\right) \\
& \cup\left(\operatorname{int}\left(\bigcap_{\beta<\alpha} \cup T_{\beta}\right)-\left(U T_{\alpha}^{\prime}\right)\right) \cup U\left\{g^{\prime}-\left(U T_{\alpha}\left(g^{\prime}\right)\right): g^{\prime} \in T_{\alpha}^{\prime}\right\}
\end{aligned}
$$

and $X$ is a countable chain condition, $X-\left(\operatorname{int}\left(\prod_{\beta<\alpha+1}^{\cap} \cup T_{\beta}\right)\right)$ has a dense first category set. Assume that $\alpha$ is limit and

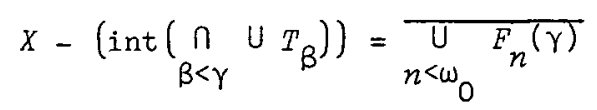

for each $\gamma<\alpha$, where $F_{n}(\gamma)$ is a nowhere dense set of $X$ for each $n<\omega_{0}$. Then clearly

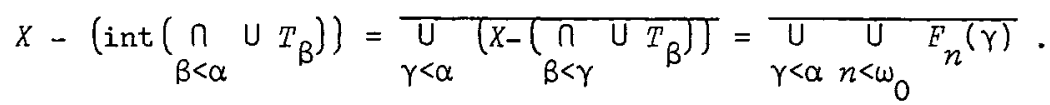

Hence $X-\left(\operatorname{int}\left(\bigcap_{B<\alpha} \cup T_{\beta}\right)\right)$ has a dense first category set. This completes the proof.

The following is a consequence of the above theorems.

COROLLARY. The following conditions are equivalent:

(1) there exists a Souslin line;

(2) there exists a connected Souslin line;

(3) there exists a Souslin tree;

(4) there exists a countable chain condition space which is not weak-separable but has a generic $\pi$-base; 
(5) there exists a locally connected countable chain condition space which is not weak-separable;

(6) there exists a countable chain condition space $X$ which is not weak-separable such that

$$
\{x \in X: X \text { is locally connected at } x\}
$$

is dense in $X$;

(7) there exists a countable chain condition space which is not weak-separable but has a $\pi$-base consisting of connected open sets.

Proof. The equivalence of (1) and (2) is due to Kunen [2], and the equivalence of (1) and (3) is due to Miller [4]. The implication of $(1) \rightarrow(4)$ follows from Theorem 1 , but the implication of $(2) \rightarrow(4)$ follows only from the remark of Theorem 1 . The implication of $(4) \rightarrow(3)$ follows from Theorem 2. The implication of $(5) \rightarrow(6) \rightarrow(7) \rightarrow(4)$ is clear. $(2) \rightarrow(5)$ also follows from Theorem 1 and its remark.

REMARK. Since the existence of a Souslin line is independent of the Zermelo-Frankel set theory with the Axiom of Choice, the existence of a locally connected countable chain condition space, which is not weakseparable, is also independent of the Zermelo-Frankel set theory with the Axiom of Choice by the corollary. But there exists a locally connected countable chain condition space which is not separable; see the next example.

EXAMPLE. Let $k$ be a cardinal such that $K>2^{\kappa} 0$. Then $I^{\kappa}$ is a countable chain condition by Engelking [1,2.3.17], where $I$ denotes the unit interval of the real line. Moreover $I^{K}$ is not separable; see Pondiczery [5] and Marczewski [3]. Furthermore, since $I$ is connected and locally connected, $I^{k}$ is locally connected. But $I^{k}$ is weak-separable.

\section{References}

[1] Ryszard Engelking, General topology (Monografie Mathematyczne, 60. PWN - Polish Scientific Publishers, Warsaw, 1977). 
[2] Kenneth Kunen, Set theory. An introduction to independence proofs (Studies in Logic and the Foundations of Mathematics, 102. North-Holland, Amsterdam, New York, Oxford, 1980).

[3] Edward Marczewski, "Séparabilité et multiplication cartésienne des espaces topologiques", Fund. Math. 34 (1947), 127-143.

[4] Edwin W. Miller, "A note on Souslin's problem", Amer. J. Math. 65 $(1943), 673-678$.

[5] E.S. Pondiczery, "Power problems in abstract spaces", Duke Math. J. 11 $(1944), 835-837$.

[6] R.M. Solovay and S. Tennenbaum, "Iterated Cohen extensions and Souslin's problem", Ann. of Math. (2) 94 (1971), 201-245.

[7] Franklin D. Tall, "Stalking the Souslin tree - a topological guide", Canad. Math. Bull. 19 (1976), 337-341.

Department of Mathematics,

Kobe University,

Nada,

Kobe 657 ,

Japan. 